\title{
Key agreement protocols for distributed secure multicast over the ring $\boldsymbol{E}_{p}^{(m)}$
}

\author{
J.-J. Climent ${ }^{1}$, J. A. López-Ramos ${ }^{2}$, P. R. Navarro ${ }^{3}$ \& L. Tortosa ${ }^{3}$ \\ ${ }^{1}$ Departament d'Estadística i Investigació Operativa, \\ Universitat d'Alacant, Spain \\ ${ }^{2}$ Departamento de Matemáticas, Universidad de Almería, Spain \\ ${ }^{3}$ Departament de Ciència de la Computació i Intel-ligència Artificial, \\ Universitat d'Alacant, Spain
}

\begin{abstract}
Protocols for authenticated key exchange allow parties within an insecure network to establish a common session key which can then be used to secure their future communication. In this paper we introduce a protocol for distributed key agreement over a noncommutative ring with a large number of noninvertible elements. This protocol uses polynomials with coefficients in the center of the ring. We also present the necessary steps for recalculating the shared secret key when a new user joins the system, or when a user leaves the system.

Keywords: secure communications, key exchange, noncommutative ring, multicast protocol.
\end{abstract}

\section{Introduction}

The classical systems of cryptography all suffer about the well-known key distribution problem. This is the problem of establishing a private channel by means of which the sender and receiver of messages can exchange the key current in use. Diffie and Hellman [1] addressed this problem in their seminal paper in 1976. The security of this protocol is based on the problem of computing discrete logarithms in the multiplicative group of a finite field.

Most of the public key cryptosystems and public key exchange protocols are based, from the point of view of its security, on the difficulty to solve some number theory problems over finite commutative algebraic structures. Some efficient 
attacks have been proposed for many of the well-known protocols in the last decades. For example, Odoni et al. [2] propose the group of matrices over a finite field as base group for Diffie-Hellman key exchange; however, this model is cryptanalyzed by Menezes and Wu [3] using eigenvalues theoretic properties.

With the main objective to avoid the attacks over commutative structures, different models have been proposed in recent years. In 2007, Cao et al. [4] present a general key exchange protocol, whose security is based on the difficulty to solve the Symmetric Decomposition Problem over a noncommutative ring, using polynomials.

Traditional communication modes have been one-to-one or unicast, and one-toall or broadcast. Among these two extremes we find multicast, the targeting of a single data stream to a select set of receivers, which may or may not include the sender. Therefore, we can say that multicasting is the ability to transmit a single stream to multiple subscribers at the same time.

There are three fundamental types of IPv4 addresses: unicast, broadcast, and multicast. A unicast address is designed to transmit a packet to a single destination. A broadcast address is used to send a datagram to an entire subnetwork. A multicast address is designed to enable the delivery of datagrams to a set of hosts that have been configured as members of a multicast group in various scattered subnetworks. Network-level IP multicast was proposed over a decade ago (see, for example, $[5,6])$.

With the main objective to avoid the attacks over commutative structures, Climent et al. [7] study the ring $\operatorname{End}\left(\mathbb{Z}_{p} \times \mathbb{Z}_{p^{2}}\right)$, for a prime $p$, and prove that it is isomorph to the ring $E_{p}$ whose elements are $2 \times 2$ matrices, with entries in the first row belonging to $\mathbb{Z}_{p}$ and the entries in the second row belonging to $\mathbb{Z}_{p^{2}}$. Using this ring and the arithmetic implemented over it, some key exchange protocols are presented in $[8,9]$, using polynomials which coefficients are elements of the center of the ring. One of these protocols based on the noncommutative ring $E_{p}$ was cryptanalyzed by Kamal and Youssef [10]. This cryptanalysis is based on the existence of a large number of invertible elements in $E_{p}$, when $p$ is large enough.

To avoid this weakness Climent et al. [11] introduce an extension $E_{p}^{(m)}$ of $E_{p}$ that maintains the main properties of $E_{p}$. In particular, it can not be embedded in a ring of matrices over a commutative ring. And most importantly, the number of noninvertible elements in $E_{p}^{(m)}$ is very large when $m$ is large compared with $p$.

The rest of the paper is organized as follows. In Section 2 we recall some properties of the ring $E_{p}^{(m)}$. In Section 3 we introduce a multicast communication protocol initially defined over any noncommutative ring $R$, based on key exchanges developed by Climent et al. [8]. The implementation of the multicast protocol is performed over the ring $E_{p}^{(m)}$, due to its characteristics that make it safe to known attacks. In Section 4 we describe the process to join or leave the group and in Section 5 we give an example for a system with 4 users. Finally, we present our main conclusions in Section 6. 


\section{The ring $E_{p}^{(m)}$}

Climent et al. [11] proved that the set

$$
\begin{aligned}
E_{p}^{(m)}=\left\{\left[a_{i j}\right] \in \operatorname{Mat}_{m \times m}(\mathbb{Z}) \mid\right. & a_{i j} \in \mathbb{Z}_{p^{i}} \text { if } i \leq j, \\
& \text { and } \left.a_{i j} \in p^{i-j} \mathbb{Z}_{p^{j}} \text { if } i>j\right\}
\end{aligned}
$$

is a noncommutative unitary ring with addition and multiplication given by

$$
\begin{aligned}
{\left[a_{i j}\right]+\left[b_{i j}\right] } & =\left[\left(a_{i j}+b_{i j}\right) \bmod p^{i}\right], \\
{\left[a_{i j}\right] \cdot\left[b_{i j}\right] } & =\left[\left(\sum_{k=1}^{m} a_{i k} b_{k j}\right) \bmod p^{i}\right],
\end{aligned}
$$

respectively. That is, the addition and multiplication of the elements of $E_{p}^{(m)}$ is analogous to the addition and multiplication of $m \times m$ matrices with entries in $\mathbb{Z}$, with the particularity that the entries in the $i$ th row are reduced modulo $p^{i}$, for $i=1,2, \ldots, m$. So, the mull matrix and the identity matrix are the additive and multiplicative identities of $E_{p}^{(m)}$. As we mentioned earlier in Section 1, the number of noninvertible elements in $E_{p}^{(m)}$ is very large when $m$ is large compared with $p$. For example, for $p=7$ and $m=2,4,8,16,32$, the number of noninvertible elements is about $26.53 \%, 46.02 \%, 70.86 \%, 91.51 \%$ and $99.28 \%$ respectively (see [11]).

Moreover, this ring is not an integral domain. So it is not a left nor a right Euclidean ring and, consequently, the ring of polynomials with coefficients in $E_{p}^{(m)}$ is not Euclidean.

The center of this ring plays an important role in the protocol that we will introduce in Section 3 and it is characterized as

$$
\begin{aligned}
& Z\left(E_{p}^{(m)}\right)=\left\{\left[a_{i j}\right] \in E_{p}^{(m)} \mid a_{i j}=0 \text { if } i \neq j,\right. \\
& \text { and } \left.a_{i i}=\sum_{r=1}^{i} p^{i-r} u_{i-r} \text { with } u_{i-r} \in \mathbb{Z}_{p}\right\} .
\end{aligned}
$$

So, $\left|Z\left(E_{p}^{(m)}\right)\right|=p^{m}$. Furthermore, $Z\left(E_{p}^{(m)}\right)$ is not an Euclidean ring.

\section{A multicast protocol over $E_{p}^{(m)}$}

The communication model we propose in this multicast protocol over $E_{p}^{(m)}$ is based on the IP multicast framework. We need to perform communications in a restricted group, where all the components (members or users) of this restricted group will manage all rekeying operations by themselves. 
Steiner et al. [12] introduce two new protocol that enhances the extension of the Diffie-Hellman key exchange for rekeying. Moreover, one of them, widely known as CLIQUES, is used for the same authors [13] for rekeying in Dynamic Peer Groups.

Before starting to describe the details of the protocol, we need to introduce the following notation for some sums:

$$
\sigma(j, 1, i)=\sum_{j=1}^{i-1} j \quad \text { and } \quad \delta(j, 1, i, l)=\sum_{\substack{j=1 \\ j \neq i-l}}^{i-1} j .
$$

So let us assume that the set of users is given by $\left\{U_{1}, U_{2}, \ldots, U_{h}\right\}$. Then, users agree to use the noncommutative ring $E_{p}^{(m)}$. Furthermore, note that if we consider $f(\mathrm{x}), g(\mathrm{x}) \in Z\left(E_{p}^{(m)}\right)[\mathrm{x}]$ and $M \in E_{p}^{(m)}$, we have that

$$
f(M)^{r} g(M)^{s}=g(M)^{r} f(M)^{s}, \quad \text { for all positive integers } r \text { and } s,
$$

although $E_{p}^{(m)}$ is not commutative. This property allows us to establish the following protocol.

Protocol 1: Let us assume that $M \in E_{p}^{(m)}$ and $K_{0}=N \in E_{p}^{(m)} \backslash Z\left(E_{p}^{(m)}\right)$ are public. Every user $U_{i}$, for $i=1,2, \ldots, h$ chooses a polynomial $f_{i}(\mathrm{x}) \in$ $Z\left(E_{p}^{(m)}\right)[\mathrm{x}]$ and a pair of positive integers $r_{i}$ and $s_{i}$. Then $\left(r_{i}, s_{i}, f_{i}(\mathrm{x})\right)$ is the private key for the user $U_{i}$.

1. User $U_{1}$ computes the element $K_{1}$ of $E_{p}^{(m)}$ given by

$$
K_{1}=f_{1}(M)^{r_{1}} K_{0} f_{1}(M)^{s_{1}} .
$$

User $U_{1}$ sends $K_{1}$ to user $U_{2}$.

2. User $U_{2}$ computes the elements $K_{2}$ and $K_{3}$ of $E_{p}^{(m)}$ given by

$$
\begin{aligned}
& K_{2}=f_{2}(M)^{r_{2}} K_{0} f_{2}(M)^{s_{2}}, \\
& K_{3}=f_{2}(M)^{r_{2}} K_{1} f_{2}(M)^{s_{2}} .
\end{aligned}
$$

User $U_{2}$ sends to user $U_{3}$ the 3 -vector of elements in $E_{p}^{(m)}$ given by

$$
\left(K_{1}, K_{2}, K_{3}\right)
$$

3. User $U_{3}$ computes the elements $K_{4}, K_{5}$ and $K_{6}$ of $E_{p}^{(m)}$ given by

$$
\begin{aligned}
& K_{4}=f_{3}(M)^{r_{3}} K_{1} f_{3}(M)^{s_{3}}, \\
& K_{5}=f_{3}(M)^{r_{3}} K_{2} f_{3}(M)^{s_{3}}, \\
& K_{6}=f_{3}(M)^{r_{3}} K_{3} f_{3}(M)^{s_{3}} .
\end{aligned}
$$


User $U_{3}$ sends to user $U_{4}$ the 4 -vector of elements in $E_{p}^{(m)}$ given by

$$
\left(K_{3}, K_{4}, K_{5}, K_{6}\right)
$$

4. In general, for $i=4,5, \ldots, h-1$, user $U_{i}$ computes the elements of $E_{p}^{(m)}$

$$
\begin{aligned}
K_{i+\delta(j, 1, i, l)} & =f_{i}(M)^{r_{i}} K_{\delta(j, 1, i, l)} f_{i}(M)^{s_{i}}, \quad \text { for } l=1,2,3, \ldots, i-1 \\
K_{i+\sigma(j, 1, i)} & =f_{i}(M)^{r_{i}} K_{\sigma(j, 1, i)} f_{i}(M)^{s_{i}}
\end{aligned}
$$

User $U_{i}$ sends to user $U_{i+1}$ the $(i+1)$-vector of elements in $E_{p}^{(m)}$ given by

$$
\begin{gathered}
\left(K_{i-1+\delta(j, 1, i, 1)}, K_{i+\delta(j, 1, i, 1)}, K_{i+\delta(j, 1, i, 2)},\right. \\
\left.\ldots, K_{i+\delta(j, 1, i, i-1)}, K_{\sigma(j, 1, i+1)}\right) .
\end{gathered}
$$

5. When user $U_{h}$ receives the $h$-vector

$$
\begin{array}{r}
\left(K_{h-2+\delta(j, 1, h-1,1)}, K_{h-1+\delta(j, 1, h-1,1)}, K_{h-1+\delta(j, 1, h-1,2)}\right) \\
\left.\ldots, K_{h-1+\delta(j, 1, h-1, h-2)}, K_{\sigma(j, 1, h)}\right) .
\end{array}
$$

he/she computes the elements of $E_{p}^{(m)}$ given by

$$
\begin{aligned}
L_{1}^{(h)} & =f_{h}(M)^{r_{h}} K_{h-2+\delta(j, 1, h-1,1)} f_{h}(M)^{s_{h}}, \\
L_{l}^{(h)} & =f_{h}(M)^{r_{h}} K_{h-1+\delta(j, 1, h-1, l-1)} f_{h}(M)^{s_{h}}, \\
& =f_{h}(M)^{r_{h}} K_{\delta(j, 1, h, l)} f_{h}(M)^{s_{h}}, \quad \text { for } l=2,3, \ldots, h-1, \\
L_{h}^{(h)} & =f_{h}(M)^{r_{h}} K_{\sigma(j, 1, h)} f_{h}(M)^{s_{h}} .
\end{aligned}
$$

User $U_{h}$ sends to every user the $(h-1)$-vector $\left(L_{1}^{(h)}, L_{2}^{(h)}, \ldots, L_{h-1}^{(h)}\right)$ of elements in $E_{p}^{(m)}$.

6. Finally, when every user $U_{i}$, for $i=1,2, \ldots, h-1$, receives the $(h-1)$ vector $\left(L_{1}^{(h)}, L_{2}^{(h)}, \ldots, L_{h-1}^{(h)}\right)$, he/she takes the $(h-i)$ th entry, $L_{h-i}^{(h)}$, and computes the element

$$
S_{i}=f_{i}(M)^{r_{i}} L_{h-i}^{(h)} f_{i}(M)^{s_{i}} .
$$

User $U_{h}$ denote by $S_{h}$ the element $L_{h}^{(h)}$, i.e., $S_{h}=L_{h}^{(h)}$.

Next theorem establishes that the shared secret by all users is $L_{h}^{(h)}$.

Theorem 1: With the notation of Protocol 1, it follows that

$$
S_{1}=S_{2}=\cdots=S_{h-1}=S_{h} .
$$


Proof: Assume that $i=1,2, \ldots, h$. From expressions (2)-(5) and (7)-(9), and taking into account expression (1), it follows that

$$
L_{h-i}^{(h)}=\left(\prod_{\substack{j=1 \\ j \neq i}}^{h} f_{j}(M)^{r_{j}}\right) K_{0}\left(\prod_{\substack{j=1 \\ j \neq i}}^{h} f_{j}(M)^{s_{j}}\right) .
$$

Now, from expressions (10) and (12) and taking into account expression (1) again, it follows that

$$
S_{i}=\left(\prod_{j=1}^{h} f_{j}(M)^{r_{j}}\right) K_{0}\left(\prod_{j=1}^{h} f_{j}(M)^{s_{j}}\right) .
$$

So, expression (11) holds.

This protocol reduces considerably the number of messages and rounds. These are exactly $h$ in both cases.

Let us remark finally that in the corresponding protocol given in [12] and [13], a simple division on a finite field would yield every power computed for every user and thus, to compromise every user's private key. In the case we are considering, this attack is not possible since the number of noninvertible elements in $E_{p}^{(m)}$ is very large when $m$ is large compared with $p$ as we stated in Section 2.

\section{Join-leave operations}

When a new user $U_{h+1}$ joins the group a rekeying is needed in order to preserve backward secrecy.

Let us assume that $U_{h}$ has stored the $h$-vector of elements in $E_{p}^{(m)}$ given by expression (6). Then the join operation will consist of the following steps:

1. User $U_{h}$ generates a new polynomial $\hat{f}_{h}(\mathrm{x}) \in Z\left(E_{p}^{(m)}\right)$ as well as two new positive integers $\hat{r}_{h}$ and $\hat{s}_{h}$. Then, he/she computes the elements of $E_{p}^{(m)}$ given by

$$
\begin{aligned}
K_{h+\delta(j, 1, h, l)} & =\hat{f}_{h}(M)^{\hat{r}_{h}} K_{\delta(j, 1, h, l)} \hat{f}_{h}(M)^{\hat{s}_{h}}, \quad \text { for } l=2,3, \ldots, h-1, \\
K_{h+\sigma(j, 1, h)} & =\hat{f}_{h}(M)^{\hat{r}_{h}} K_{\sigma(j, 1, h)} \hat{f}_{h}(M)^{\hat{s}_{h}} .
\end{aligned}
$$

User $U_{h}$ sends to the new user $U_{h+1}$ the $(h+1)$-vector

$$
\begin{gathered}
\left(K_{h-1+\delta(j, 1, h, 1)}, K_{h+\delta(j, 1, h, 1)}, K_{h+\delta(j, 1, h, 2)},\right. \\
\left.\ldots, K_{h+\delta(j, 1, h, h-1)}, K_{\sigma(j, 1, h+1)}\right) .
\end{gathered}
$$

2. User $U_{h+1}$ acts as previously user $U_{h}$ did, and computes the elements of $E_{p}^{(m)}$ given by

$$
L_{1}^{(h+1)}=f_{h+1}(M)^{r_{h+1}} K_{h-1+\delta(j, 1, h, 1)} f_{h+1}(M)^{s_{h+1}},
$$




$$
\begin{aligned}
L_{l}^{(h+1)} & =f_{h+1}(M)^{r_{h+1}} K_{h+\delta(j, 1, h, l-1)} f_{h+1}(M)^{s_{h+1}}, \\
& =f_{h+1}(M)^{r_{h+1}} K_{\delta(j, 1, h+1, l)} f_{h+1}(M)^{s_{h+1}}, \quad \text { for } l=2,3, \ldots, h, \\
L_{h+1}^{(h+1)} & =f_{h+1}(M)^{r_{h+1}} K_{\sigma(j, 1, h+1)} f_{h+1}(M)^{s_{h+1}} .
\end{aligned}
$$

3. User $U_{h+1}$ sends to every user the $h$-vector of elements of $E_{p}^{(m)}$ given by

$$
\left(L_{1}^{(h+1)}, L_{2}^{(h+1)}, \ldots, L_{h}^{(h+1)}\right) .
$$

4. Finally, when every user $U_{i}$, for $i=1,2, \ldots, h$, receives the $h$-vector given by expression (13), he/she takes the $(h+1-i)$ th entry, $L_{h+1-i}^{(h+1)}$, and computes the new element $\hat{S}_{i}$ as

$$
\hat{S}_{i}=f_{i}(M)^{r_{i}} L_{h+1-i}^{(h+1)} f_{i}(M)^{s_{i}}, \quad \text { for } i=1,2, \ldots, h .
$$

User $U_{h+1}$ denote by $\hat{S}_{h}$ the element $L_{h+1}^{(h+1)}$, i.e., $\hat{S}_{h+1}=L_{h+1}^{(h+1)}$. Now, Theorem 1 ensures that the shared secret by all users is

$$
\hat{S}_{1}=\hat{S}_{2}=\cdots=\hat{S}_{h}=\hat{S}_{h+1} .
$$

Assume again that the system consists of $h$ users: $U_{1}, U_{2}, \ldots, U_{h}$. if user $U_{i}$, for some $i=1,2, \ldots, h-1$, decides to leave the system, a rekeying is also needed in order to preserve forward secrecy.

Recall that user $U_{h}$ has the $h$-vector of elements in $E_{p}^{(m)}$ given by expression (6). As in the join operation, user $U_{h}$ generates two new positive integers $\hat{r}_{h}$ and $\hat{s}_{h}$, as well as a new polynomial $\hat{f}_{h}(\mathrm{x}) \in Z\left(E_{p}^{(m)}\right)$. Then, the elements of $E_{p}^{(m)}$ are computed by

$$
\begin{aligned}
L_{1}^{(h)} & =\hat{f}_{h}(M)^{\hat{r}_{h}} K_{h-2+\delta(j, 1, h-1,1)} \hat{f}_{h}(M)^{\hat{s}_{h}}, \\
L_{l}^{(h)} & =\hat{f}_{h}(M)^{\hat{r}_{h}} K_{h-1+\delta(j, 1, h-1, l-1)} \hat{f}_{h}(M)^{\hat{s}_{h}}, \\
& =\hat{f}_{h}(M)^{\hat{r}_{h}} K_{\delta(j, 1, h, l)} \hat{f}_{h}(M)^{\hat{s}_{h}}, \quad \text { for } l=2,3, \ldots, h-1, \\
L_{h}^{(h)} & =\hat{f}_{h}(M)^{\hat{r}_{h}} K_{\sigma(j, 1, h)} \hat{f}_{h}(M)^{\hat{s}_{h}} .
\end{aligned}
$$

That is, expressions (7)-(9) are used to compute $L_{l}^{(h)}$, but replacing $f_{l}(M), r_{l}$ and $s_{l}$, by $\hat{f}_{l}(M), \hat{r}_{l}$ and $\hat{s}_{l}$, respectively, for $l=1,2, \ldots, h$. Note that it is not necessary to compute $L_{h-i}^{(h)}$, because user $U_{i}$ leaves the group.

Finally, user $U_{h}$ sends to all users, except to user $U_{i}$, the $(h-1)$-vector

$$
\left(L_{1}^{(h)}, L_{2}^{(h)}, \ldots, L_{h-1}^{(h)}\right)
$$

and each user computes the new shared secret key as in step 6 of Protocol 1.

In case user $U_{h}$ decides to leave the system, then user $U_{h-1}$ changes his/her private key and acts as $U_{h}$. 


\section{Example}

In this example we show how to share a secret in a system with $h=4$ uses using Protocol 1.

Assume that $p=2$ and $m=5$ and consider the public elements $M \in E_{2}^{(5)}$ and $N \in E_{2}^{(5)} \backslash Z\left(E_{2}^{(5)}\right)$ given by

$$
M=\left[\begin{array}{ccccc}
1 & 0 & 0 & 0 & 1 \\
0 & 0 & 0 & 1 & 0 \\
0 & 2 & 3 & 2 & 7 \\
8 & 0 & 2 & 14 & 0 \\
0 & 8 & 4 & 2 & 23
\end{array}\right] \quad \text { and } \quad N=\left[\begin{array}{ccccc}
1 & 1 & 0 & 1 & 1 \\
2 & 0 & 0 & 2 & 3 \\
4 & 0 & 5 & 1 & 2 \\
0 & 0 & 0 & 7 & 15 \\
0 & 8 & 0 & 0 & 5
\end{array}\right]
$$

For some polynomials $f_{1}(\mathrm{x}), f_{2}(\mathrm{x}), f_{3}(\mathrm{x}), f_{4}(\mathrm{x}) \in Z\left(E_{2}^{(5)}\right)[\mathrm{x}]$ we have obtained the following elements:

$$
\begin{aligned}
f_{1}(M) & =\left[\begin{array}{ccccc}
1 & 0 & 0 & 0 & 1 \\
0 & 3 & 0 & 0 & 2 \\
0 & 4 & 1 & 6 & 5 \\
0 & 8 & 12 & 7 & 14 \\
16 & 16 & 16 & 20 & 21
\end{array}\right], f_{2}(M)=\left[\begin{array}{ccccc}
1 & 0 & 0 & 0 & 1 \\
0 & 1 & 0 & 0 & 2 \\
0 & 0 & 1 & 6 & 7 \\
0 & 0 & 0 & 13 & 6 \\
16 & 16 & 24 & 8 & 13
\end{array}\right], \\
f_{3}(M) & =\left[\begin{array}{ccccc}
1 & 0 & 0 & 0 & 0 \\
0 & 0 & 2 & 0 & 0 \\
0 & 2 & 7 & 6 & 4 \\
8 & 4 & 6 & 4 & 6 \\
16 & 16 & 20 & 30 & 27
\end{array}\right], f_{4}(M)=\left[\begin{array}{ccccc}
1 & 0 & 0 & 0 & 0 \\
0 & 2 & 0 & 3 & 2 \\
0 & 2 & 5 & 4 & 6 \\
8 & 8 & 2 & 4 & 6 \\
16 & 8 & 28 & 26 & 29
\end{array}\right]
\end{aligned}
$$

Assume that $K_{0}=N$ and consider

$$
\left(r_{1}, r_{2}, r_{3}, r_{4}\right)=(10,5,6,11) \quad \text { and } \quad\left(s_{1}, s_{2}, s_{3}, s_{4}\right)=(5,14,16,12) .
$$

Recall that the $\left(r_{i}, s_{i}, f_{i}(M)\right)$ is the private key of user $U_{i}$ for $i=1,2,3,4$.

User $U_{1}$ computes the element

$$
K_{1}=f_{1}(M)^{r_{1}} K_{0} f_{1}(M)^{s_{1}}=\left[\begin{array}{ccccc}
1 & 1 & 0 & 1 & 0 \\
2 & 0 & 0 & 2 & 1 \\
4 & 4 & 1 & 1 & 7 \\
0 & 8 & 4 & 13 & 9 \\
16 & 8 & 0 & 4 & 9
\end{array}\right]
$$

User $U_{1}$ sends $K_{1}$ to user $U_{2}$.

User $U_{2}$ computes the elements

$$
\begin{aligned}
K_{2}=f_{2}(M)^{r_{2}} K_{0} f_{2}(M)^{s_{2}}= & {\left[\begin{array}{ccccc}
1 & 1 & 0 & 1 & 0 \\
2 & 0 & 0 & 2 & 1 \\
4 & 0 & 5 & 7 & 1 \\
0 & 0 & 0 & 3 & 5 \\
16 & 24 & 8 & 0 & 9
\end{array}\right], } \\
K_{3}=f_{2}(M)^{r_{2}} K_{1} f_{2}(M)^{s_{2}}= & {\left[\begin{array}{ccccc}
1 & 1 & 0 & 1 & 1 \\
2 & 0 & 0 & 2 & 3 \\
4 & 4 & 1 & 7 & 6 \\
0 & 8 & 4 & 9 & 15 \\
0 & 24 & 8 & 4 & 5
\end{array}\right] . }
\end{aligned}
$$


User $U_{2}$ sends to user $U_{3}$ the 3 -vector $\left(K_{1}, K_{2}, K_{3}\right)$.

User $U_{3}$ computes

$$
\begin{aligned}
K_{4}=f_{3}(M)^{r_{3}} K_{1} f_{3}(M)^{s_{3}}= & {\left[\begin{array}{ccccc}
1 & 0 & 0 & 0 & 0 \\
0 & 0 & 2 & 0 & 2 \\
0 & 2 & 3 & 0 & 1 \\
8 & 4 & 6 & 12 & 0 \\
16 & 0 & 12 & 6 & 27
\end{array}\right], } \\
K_{5}=f_{3}(M)^{r_{3}} K_{2} f_{3}(M)^{s_{3}}= & {\left[\begin{array}{ccccc}
1 & 0 & 0 & 0 & 0 \\
0 & 0 & 2 & 0 & 2 \\
0 & 2 & 3 & 4 & 7 \\
8 & 4 & 6 & 4 & 12 \\
16 & 0 & 28 & 6 & 11
\end{array}\right], } \\
K_{6}=f_{3}(M)^{r_{3}} K_{3} f_{3}(M)^{s_{3}} & =\left[\begin{array}{ccccc}
1 & 0 & 0 & 0 & 1 \\
0 & 0 & 2 & 0 & 0 \\
0 & 2 & 3 & 6 & 0 \\
8 & 4 & 6 & 8 & 6 \\
0 & 16 & 4 & 14 & 7
\end{array}\right] .
\end{aligned}
$$

User $U_{3}$ sends to user $U_{4}$ the 4 -vector $\left(K_{3}, K_{4}, K_{5}, K_{6}\right)$.

User $U_{4}$ computes the elements

$$
\begin{aligned}
L_{1}^{(4)}=f_{4}(M)^{r_{4}} K_{3} f_{4}(M)^{s_{4}}= & {\left[\begin{array}{ccccc}
1 & 0 & 0 & 0 & 1 \\
0 & 0 & 2 & 0 & 0 \\
0 & 2 & 3 & 2 & 6 \\
8 & 4 & 6 & 0 & 2 \\
0 & 16 & 20 & 14 & 7
\end{array}\right], } \\
L_{2}^{(4)}=f_{4}(M)^{r_{4}} K_{4} f_{4}(M)^{s_{4}}= & {\left[\begin{array}{ccccc}
1 & 0 & 0 & 0 & 0 \\
0 & 0 & 2 & 0 & 2 \\
0 & 2 & 3 & 4 & 7 \\
8 & 4 & 6 & 4 & 12 \\
16 & 0 & 28 & 6 & 27
\end{array}\right], } \\
L_{3}^{(4)}=f_{4}(M)^{r_{4}} K_{5} f_{4}(M)^{s_{4}}= & {\left[\begin{array}{ccccc}
1 & 0 & 0 & 0 & 0 \\
0 & 0 & 2 & 0 & 2 \\
0 & 2 & 3 & 0 & 5 \\
8 & 4 & 6 & 12 & 8 \\
16 & 0 & 12 & 6 & 11
\end{array}\right], } \\
L_{4}^{(4)}=f_{4}(M)^{r_{4}} K_{6} f_{4}(M)^{s_{4}} & =\left[\begin{array}{ccccc}
1 & 0 & 0 & 0 & 1 \\
0 & 0 & 2 & 0 & 0 \\
0 & 2 & 3 & 2 & 6 \\
8 & 4 & 6 & 0 & 2 \\
0 & 16 & 20 & 14 & 23
\end{array}\right] .
\end{aligned}
$$

Let $S_{4}=L_{4}^{(4)}$.

Now, user $U_{4}$ sends to users $U_{1}, U_{2}$ and $U_{3}$ the 3 -vector $\left(L_{1}^{(4)}, L_{2}^{(4)}, L_{3}^{(4)}\right)$.

User $U_{1}$ uses $L_{3}^{(4)}$ to compute $S_{1}=f_{1}(M)^{r_{1}} L_{3}^{(4)} f_{1}(M)^{s_{1}}$.

User $U_{2}$ uses $L_{2}^{(4)}$ to compute $S_{2}=f_{2}(M)^{r_{2}} L_{2}^{(4)} f_{2}(M)^{s_{2}}$.

Finally, user $U_{3}$ uses $L_{1}^{(4)}$ to compute $S_{3}=f_{3}(M)^{r_{3}} L_{1}^{(4)} f_{3}(M)^{s_{3}}$.

Now, as we established in Theorem 1, $S_{1}=S_{2}=S_{3}=S_{4}$. 
Assume now that a new user, $U_{5}$, wants to join the above system.

User $U_{4}$ generates a new private key $\left(\hat{r}_{4}, \hat{s}_{4}, \hat{f}_{4}(M)\right)$ with $\hat{r}_{4}=7, \hat{s}_{4}=4$ and

$$
\hat{f}_{4}(M)=\left[\begin{array}{ccccc}
1 & 0 & 0 & 0 & 1 \\
0 & 3 & 2 & 3 & 0 \\
0 & 0 & 7 & 4 & 5 \\
0 & 4 & 12 & 5 & 14 \\
16 & 8 & 0 & 4 & 23
\end{array}\right]
$$

User $U_{4}$ computes

$$
\begin{aligned}
K_{7}=\hat{f}_{4}(M)^{\hat{r}_{4}} K_{3} \hat{f}_{4}(M)^{\hat{s}_{4}}= & {\left[\begin{array}{ccccc}
1 & 1 & 0 & 1 & 0 \\
2 & 0 & 2 & 3 & 2 \\
4 & 4 & 7 & 5 & 5 \\
8 & 8 & 8 & 5 & 13 \\
0 & 24 & 8 & 8 & 23
\end{array}\right], } \\
K_{8}=\hat{f}_{4}(M)^{\hat{r}_{4}} K_{4} \hat{f}_{4}(M)^{\hat{s}_{4}}= & {\left[\begin{array}{ccccc}
1 & 0 & 0 & 1 & 1 \\
0 & 0 & 2 & 0 & 0 \\
0 & 6 & 1 & 2 & 4 \\
8 & 12 & 2 & 8 & 10 \\
0 & 16 & 12 & 10 & 5
\end{array}\right], } \\
K_{9}=\hat{f}_{4}(M)^{\hat{r}_{4}} K_{5} \hat{f}_{4}(M)^{\hat{s}_{4}}= & {\left[\begin{array}{ccccc}
1 & 0 & 0 & 0 & 1 \\
0 & 0 & 2 & 0 & 0 \\
0 & 6 & 1 & 6 & 6 \\
8 & 12 & 2 & 0 & 14 \\
0 & 16 & 28 & 10 & 5
\end{array}\right], } \\
K_{10}=\hat{f}_{4}(M)^{\hat{r}_{4}} K_{6} \hat{f}_{4}(M)^{\hat{s}_{4}}= & {\left[\begin{array}{ccccc}
1 & 0 & 0 & 0 & 0 \\
0 & 0 & 2 & 0 & 2 \\
0 & 6 & 1 & 4 & 1 \\
8 & 12 & 2 & 12 & 12 \\
16 & 0 & 20 & 18 & 1
\end{array}\right] . }
\end{aligned}
$$

User $U_{4}$ sends to user $U_{5}$ the 5 -vector $\left(K_{6}, K_{7}, K_{8}, K_{9}, K_{10}\right)$.

User $U_{5}$ generates a private key $\left(r_{5}, s_{5}, f_{5}(M)\right)$ with $r_{5}=4, s_{5}=10$ and

$$
f_{5}(M)=\left[\begin{array}{ccccc}
1 & 0 & 0 & 0 & 1 \\
0 & 0 & 0 & 1 & 0 \\
0 & 6 & 5 & 6 & 7 \\
8 & 8 & 6 & 6 & 4 \\
0 & 8 & 28 & 6 & 17
\end{array}\right]
$$

User $U_{5}$ computes the elements

$$
\begin{aligned}
L_{1}^{(5)}=f_{5}(M)^{r_{5}} K_{6} f_{5}(M)^{s_{5}}= & {\left[\begin{array}{ccccc}
1 & 0 & 0 & 0 & 1 \\
0 & 0 & 2 & 0 & 0 \\
0 & 2 & 3 & 2 & 6 \\
8 & 4 & 6 & 0 & 2 \\
0 & 16 & 20 & 14 & 23
\end{array}\right], } \\
L_{2}^{(5)}=f_{5}(M)^{r_{5}} K_{7} f_{5}(M)^{s_{5}}= & {\left[\begin{array}{ccccc}
1 & 0 & 0 & 0 & 0 \\
0 & 0 & 2 & 0 & 2 \\
0 & 6 & 1 & 0 & 3 \\
8 & 12 & 2 & 4 & 0 \\
16 & 0 & 4 & 18 & 17
\end{array}\right], }
\end{aligned}
$$




$$
\begin{aligned}
L_{3}^{(5)}=f_{5}(M)^{r_{5}} K_{8} f_{5}(M)^{s_{5}}= & {\left[\begin{array}{ccccc}
1 & 0 & 0 & 0 & 1 \\
0 & 0 & 2 & 0 & 0 \\
0 & 6 & 1 & 6 & 6 \\
8 & 12 & 2 & 0 & 14 \\
0 & 16 & 28 & 10 & 21
\end{array}\right], } \\
L_{4}^{(5)}=f_{5}(M)^{r_{5}} K_{9} f_{5}(M)^{s_{5}}= & {\left[\begin{array}{ccccc}
1 & 0 & 0 & 0 & 1 \\
0 & 0 & 2 & 0 & 0 \\
0 & 6 & 1 & 2 & 0 \\
8 & 12 & 2 & 8 & 2 \\
0 & 16 & 12 & 10 & 21
\end{array}\right], } \\
L_{5}^{(5)}=f_{5}(M)^{r_{5}} K_{10} f_{5}(M)^{s_{5}} & =\left[\begin{array}{ccccc}
1 & 0 & 0 & 0 & 0 \\
0 & 0 & 2 & 0 & 2 \\
0 & 6 & 1 & 0 & 3 \\
8 & 12 & 2 & 4 & 0 \\
16 & 0 & 4 & 18 & 1
\end{array}\right] .
\end{aligned}
$$

Let $S_{5}=L_{5}^{(5)}$.

User $U_{5}$ sends to users $U_{1}, U_{2}, U_{3}$ and $U_{4}$ the 4 -vector $\left(L_{1}^{(5)}, L_{2}^{(5)}, L_{3}^{(5)}, L_{4}^{(5)}\right)$. User $U_{1}$ uses $L_{4}^{(5)}$ to compute $S_{1}=f_{1}(M)^{r_{1}} L_{4}^{(5)} f_{1}(M)^{s_{1}}$.

User $U_{2}$ uses $L_{3}^{(5)}$ to compute $S_{2}=f_{2}(M)^{r_{2}} L_{3}^{(5)} f_{2}(M)^{s_{2}}$. User $U_{3}$ uses $L_{2}^{(5)}$ to compute $S_{3}=f_{3}(M)^{r_{3}} L_{2}^{(5)} f_{3}(M)^{s_{3}}$.

Finally, user $U_{4}$ uses $L_{1}^{(5)}$ to compute $S_{4}=f_{4}(M)^{r_{4}} L_{1}^{(5)} f_{4}(M)^{s_{4}}$.

Now, as we established in Theorem 1, $S_{1}=S_{2}=S_{3}=S_{4}=S_{5}$.

\section{Conclusions}

We introduce a key agreement protocol for secure communications based over a noncommutative ring with a large number of noninvertible elements. The protocol shows to be efficient for large audiences, making it applicable nowadays for widely extended secure multicast communications and allows users to join or leave the communication group preserving forward and backward secrecy in an efficient way.

\section{Acknowledgements}

The work of the first author was partially supported by Spanish grant MTM201124858 of the Ministerio de Economía y Competitividad of the Gobierno de España. The work of the second author was partially supported by the grant FQM 0211 of the Junta de Andalucía.

\section{References}

[1] Diffie, W.D. \& Hellman, M.E., New directions in cryptography. IEEE Transactions on Information Theory, 22(6), pp. 644-654, 1976. 
[2] Odoni, R.W.K., Varadharajan, V. \& Sanders, P.W., Public key distribution in matrix rings. Electronics Letters, 20, pp. 386-387, 1984.

[3] Menezes, A.J. \& Wu, Y.H., The discrete logarithm problem in $G L(n, q)$. Ars Combinatoria, 47, pp. 23-32, 1997.

[4] Cao, Z., Dong, X. \& Wang, L., New public key cryptosystems using polynomials over non-commutative rings. Cryptology ePrint Archive, Report 2007/007, 2007. http: / / eprint.iacr.org/.

[5] Deering, S.E. \& Cheriton, D.R., Multicast routing in datagram internetworks and extended LANs. ACM Transactions on Computer Systems, 8(2), pp. 85110, 1990.

[6] Deering, S.E., Estrin, D.L., Farinacci, D., Jacobson, V., Liu, C.G. \& Wei, L., The PIM architecture for wide-area multicast routing. IEEE/ACM Transactions on Networking, 4(2), pp. 153-162, 1996.

[7] Climent, J.J., Navarro, P.R. \& Tortosa, L., On the arithmetic of the endomorphisms ring $\operatorname{End}\left(\mathbb{Z}_{p} \times \mathbb{Z}_{p^{2}}\right)$. Applicable Algebra in Engineering, Communication and Computing, 22(2), pp. 91-108, 2011.

[8] Climent, J.J., Navarro, P.R. \& Tortosa, L., Key exchange protocols over noncommutative rings. The case $\operatorname{End}\left(\mathbb{Z}_{p} \times \mathbb{Z}_{p^{2}}\right)$. Proceedings of the 11th International Conference on Computational and Mathematical Methods in Science and Engineering (CMMSE 2011), ed. J. Vigo Aguiar, pp. 357-364, 2011.

[9] Climent, J.J., Navarro, P.R. \& Tortosa, L., Key exchange protocols over noncommutative rings. The case of $\operatorname{End}\left(\mathbb{Z}_{p} \times \mathbb{Z}_{p^{2}}\right)$. International Journal of Computer Mathematics, 89(13-14), pp. 1753-1763, 2012.

[10] Kamal, A.A. \& Youssef, A.M., Cryptanalysis of a key exchange protocol based on the endomorphisms ring $\operatorname{End}\left(\mathbb{Z}_{p} \times \mathbb{Z}_{p}^{2}\right)$. Applicable Algebra in Engineering, Communication and Computing, 23(3-4), pp. 143-149, 2012.

[11] Climent, J.J., Navarro, P.R. \& Tortosa, L., An extension of the noncommutative Bergman's ring with a large number of noninvertible elements. Submitted, 2013.

[12] Steiner, M., Tsudik, G. \& Waidner, M., Diffie-Hellman key distribution extended to group communication. Proceedings of the 3rd ACM Conference on Computer and Communications Security, ACM: New York, NY, pp. 3137, 1996.

[13] Steiner, M., Tsudik, G. \& Waidner, M., Key agreement in dynamic peer groups. IEEE Transactions of Parallel and Distributed Systems, 11(8), pp. 769-780, 2000. 